\title{
Tumor, The Formation of Amino Acid Imbalances and Metabolic Processes in the Background of Malignant Growth: The Levels and Nature of the Interaction
}

\author{
L Nefyodov $^{1 *}$ and P Karavay ${ }^{2}$ \\ ${ }^{1}$ Department of Biochemistry, Yanka Kupala State University of Grodno, Belarus \\ ${ }^{2}$ Department of Hyperbaric oxygenation, Grodno Regional Clinical Hospital, Belarus
}

*Corresponding author: L Nefyodov, Department of Biochemistry, Yanka Kupala State University of Grodno, Orzeszkowej 22, 230023 Grodno, Belarus

\begin{abstract}
A mini-review of the laws governing the formation of an amino acid fund during the development of cancer from the standpoint of competition between the tumor and the body for common substrates.
\end{abstract}

Keywords: Free Amino Acids; Oncology; Regulatory Effects

\section{Introduction}

The appearance of a malignant neoplasm is accompanied by changes in the intermediate metabolism, which manifest themselves at the level of the whole organism, individual organs, tissues, cells and enzymatic reactions. These changes are determined primarily by the properties of the tumor as a structure consisting of relatively poorly differentiated cells, characterized by unregulated growth, metastasis and necrosis [1-11]. Active tumor growth against the background of the relative reduction of individual chemical reactions (in particular, low enzyme activity and the rate of enzymatic amino acid metabolism) and the lack of perfect regulatory mechanisms to maintain the metabolic balance in malignant cells lead to a competitive relationship between the tumor and the host organism in metabolism and energy. These relationships are manifested in the ability of tumor cells to use the plastic material of the tumor carrier for the generation of energy and their own growth. Thereby, a high activity of metabolic processes is ensured in the tumor, in particular, accompanied by the formation of a significant amount of energy. Thus, the substrate provision of tumor cells becomes the limiting factor of their growth. This is particularly pronounced in cases where compounds that are absolutely or relatively essential (for example, some amino acids, essential lipids) for a macroorganism are used as an energy or plastic material. In addition, as is well known, a significant amount of cofactors of key enzymatic reactions (vitamins, microelements) are also irreplaceable nutrient factors to varying degrees. He degree of indispensability of the above compounds against the background of malignant growth, thus, increases, inducing the formation of their functional deficiency both in the body of the tumor carrier and in the tumor itself. Obviously, with their additional exogenous intake, the elimination of this deficiency will be determined by the activity of the transport systems of such compounds into host cells or tumors. Therefore, for example, anorexia at the level of the microorganism, on the one hand, and the ability of the tumor to actively "capture" endogenous compounds and nutrients, on the other hand, should be considered adaptive responses that provide in these conditions the principle of maximum survival of each system. Such a kind of "metabolic competition" of a tumor and a macroorganism significantly limits the possibilities of metabolic correction and treatment of cancer patients in cases where it is associated with the need to introduce additional quantities of biologically active compounds of natural origin. The next most important element that determines the tumor-tumor carrier relationship at the biochemical level is the phase growth of the tumor, in particular, periods of massive necrosis of cancer cells, accompanied by the appearance in the host fluids and tissues of significant amounts of a wide range of non-oxidized products and highly active prooxidants that induce a redox potential and metabolic imbalance in normal cells. The described trends in cancer diseases are especially pronounced in 
the metabolism of amino acids. Our particular interest in studying the patterns of formation of a free amino acid pool is justified by the fact that malignant growth:

a) Against the background of anorexia, it causes quantitative insufficiency of essential and relatively essential amino acids in the body, simultaneously, as a result of the activation of the degradation processes of endogenous proteins and amino acids, inducing a negative nitrogen balance;

b) Violates the processes of absorption and transport of free amino acids;

c) Shifting the redox potential of cells disrupts the relationship between anabolic and catabolic reactions in the exchange of amino acids;

d) The toxic effect of the decay products of the tumor is blocked by amino acids directly interacting with them (cystine, lysine) or their derivatives (glutathione), which leads to a deficiency of these compounds;

e) Changes the ratio and activity of metabolic processes associated with intermediate metabolism of amino acids (glycolysis, gluconeogenesis), as well as the processes of formation and energy consumption;

f) In addition, as mentioned above, amino acids and their derivatives are natural regulators of the activity of the processes of proliferation, differentiation and apoptosis of malignant cells [1$11]$.

Amino acids and their derivatives are mostly universal natural regulators and endogenous modifiers of biological reactions. However, numerous biological properties of these compounds as drugs were used for correction of deficiencies or realization of pharmacological and immediate metabolic effects, disregarding a regulatory action. Amino acid profile indices that allow early detection of diseases, which would provide time for intervention before irreversible damage occurred, are being created. Thus, amino acid profiles represent biomarkers for diseases or deviations from a normal state of health. Our array technology will play an important role in metabolomics in biomarker discovery, clinical medicine, including cancer as well as, at other stages of drug discovery and development (for example, target discovery, mechanism of action or predicting toxicity) [1-7]. Changes in amino acid pool in liquids and tissues of patients specifically characterize development of cancer. Correction of the intermediate metabolic changes in cancer can be reached by the use of certain amino acids or their combinations [810]. To understand the metabolic processes and vital functions of the regulatory effect of amino acids which manifests itself under natural or near concentrations of these compounds in body fluids and tissues. It is obvious that the effective use of L-amino acids or their derivatives for metabolic correction and directional changes in metabolism under pathological or extreme conditions is limited by insufficient accumulation of information about the key mechanisms of regulatory effects of the compounds tested at concentrations comparable to their physiological (endogenous) levels [1-13].
Amino acids and their derivatives are mostly universal natural regulators and endogenous modifiers of biological reactions. However, numerous biological properties of these compounds as drugs were used for correction of deficiencies or realization of pharmacological and immediate metabolic effects, disregarding a regulatory action. Amino acid profile indices that allow early detection of diseases, which would provide time for intervention before irreversible damage occurred, are being created. Thus, amino acid profiles represent biomarkers for diseases or deviations from a normal state of health. Our array technology will play an important role in metabolomics in biomarker discovery, clinical medicine, including cancer as well as, at other stages of drug discovery and development (for example, target discovery, mechanism of action or predicting toxicity) [1-15]. Changes in amino acid pool in liquids and tissues of patients specifically characterize development of cancer. Correction of the intermediate metabolic changes in cancer can be reached by the use of certain amino acids or their combinations [7-8].

At present, there are the following main lines in exploitation of biochemical (metabolic) properties of amino acids and their derivatives in clinical practice. Use of amino acids or multicomponent mixtures of amino acids (mainly, essential elements combined with vitamins and trace elements) for replacement therapy or deficiency of essential nutrients and proteins. Use of drugs containing individual amino acids or their compositions, designed on the basis of their additive functional and metabolic action, which "exploits" pharmacological activity (effects of activation of redox processes, reactions of energy metabolism and neutralization of xenobiotics compounds) of this class [4-9]. However, the use of certain levels of L-amino acids or their compositions or their deficiency implements direct pharmacological effects that practically ignore their regulatory effects on metabolic processes and key metabolic reactions. To understand the metabolic processes and vital functions of the regulatory effect of amino acids which manifests itself under natural or near concentrations of these compounds in body fluids and tissues. It is obvious that the effective use of L-amino acids or their derivatives for metabolic correction and directional changes in metabolism under pathological or extreme conditions is limited by insufficient accumulation of information about the key mechanisms of regulatory effects of the compounds tested at concentrations comparable to their physiological (endogenous) levels [1-9].

\section{References}

1. Nefyodov L (1996) Amino Acids and Their Derivatives (chemistry, biochemistry, pharmacology, medicine), Proc of Internat. Symp Grodno, USA, pp. 125.

2. Nefyodov L, Karavay P, Karavay N (2014) Regulatory action of free amino acids and development on the basis of highly of substances infusion solutions with pathogenetic deterministic composition. Laboratory diagnosis Eastern Europe 3: 111-115.

3. Lubec C, (1990) Amino Acids (Chemistry, Biology, Medicine) Eskom, New York, USA, pp. 1196.

4. http://www.nil.grsu.by/index.php?page=index. 
5. Nefyodov L, Uglyanica KN, Smirnov VY, Doroshenko YM, Fomin KA, et al. (1996) Amino acids and their derivatives in tumour tissue from patients with breast cancer treated with Ukrain. Part VI. Drugs Exp Clin Res 22: 155-177.

6. Nefyodov LI, Uglyanica KN, Smirnov VY, Doroshenko YM, Fomin KA, et al. (1996) Amino acids and their derivatives in blood plasma of patients with breast cancer treated with Ukrain. Part V. Drugs Exp Clin Res 22: 159-161.

7. Nefyodov L (2000) VI Ordinary General Assembly Society of Biochemistry. Proc of Internat Symp Grodno, USA pp. 225.

8. Nefyodov L (2001) Amino acids and their derivatives in biology and medicine. Proc of Internat Symp Grodno, USA pp. 124.

9. Nefyodov L (2001) Target-oriented regulation of metabolic equilibrium by amino acids and strategy of their application as drugs with directional effects. XXXVII Zjazd Polskiego towarzystwa biochemicznego, Torun, UK pp. 327.
10. Ihata Y, Miyagi E, Numazaki R (2013) Amino acid profile index for early detection of endometrial cancer: verification as a novel diagnostic marker, Int J Clin Oncol 9: 364-372.

11. Fafournoux P, Bruhat A, Jousse C (2000) Amino acid regulation of gene expression. BioChem J 351: 1-12.

12. Meijer A (2003) Amino acids as regulators and components of nonproteinogenic pathways. J Nutr 6: 2057S-2062S.

13. Bruhat A, Cherasse Y, Chaveroux C, Maurin AC, Jousse C, et al. (2009) Amino acids as regulators of gene expression in mammals: molecular mechanisms. Biofactors 35: 249-357.

14. Ananieva E (2015) Targeting amino acid metabolism in cancer growth and anti-tumor immune response. World J Biol Chem 6: 281-289.

15. Ma Hong, Hasim A, Mamtimin B, Kong B, Zhang HP, et al. (2014) Plasma free amino acid profiling of esophageal cancer using high-performance liquid chromatography spectroscopy. World J Gastroenterol 20: 86538659.

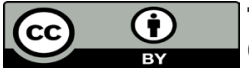

This work is licensed under Creative Commons Attribution 4.0 License

To Submit Your Article Click Here:

Submit Article
DOI: 10.32474/OAJOM.2019.03.000161

\section{Open Access Journal of Oncology} and Medicine

\section{OAJOM}

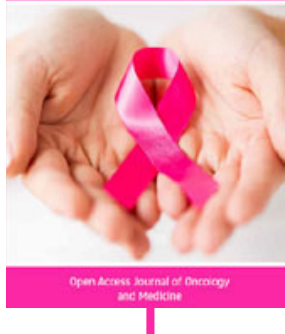

\section{Assets of Publishing with us}

- Global archiving of articles

- Immediate, unrestricted online access

- Rigorous Peer Review Process

- Authors Retain Copyrights

- Unique DOI for all articles 